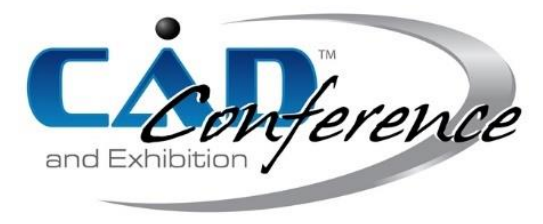

Title:

\title{
Island Scanning Path-Patten Optimization for Residual Distortion Control in Metal Additive Manufacturing
}

\section{Authors:}

Shuzhi Xu, shuzhi@ualberta.ca, University of Alberta

Jiaqi Huang, jiaqih@mail.sdu.edu.cn, Shandong University

Jikai Liu, jikai_liu@sdu.edu.cn, Shandong University

Yongsheng Ma, yongsheng.ma@ualberta.ca, University of Alberta

\section{Keywords:}

Metal additive manufacturing, path optimization, inherent strain method, voxelization

DOI: 10.14733/cadconfP.2021.66-72

\section{Introduction:}

Additive manufacturing (AM) is the fastest-growing segment of manufacturing technologies in past decades. The end-use products fabricated by the AM are increasingly being used in many industries like aerospace, automotive, machinery, and biomedical [1-6]. This concept has been developed for different materials like plastic and metal, with an adaptation of the process [7]. Among processes, powder bed fusion has the capability to build metal objects with complex geometries, and thus has captured the interest of the researchers and industry experts [8]. During the manufacturing process: a source of thermal energy (laser) moves along a planned trajectory (0), and meanwhile selectively fuses the regularly distributed powder. Then the material solidifies from cooling, and a new layer of powder is spread across the previous layer. Further layers are fused and added until the entire model is created.

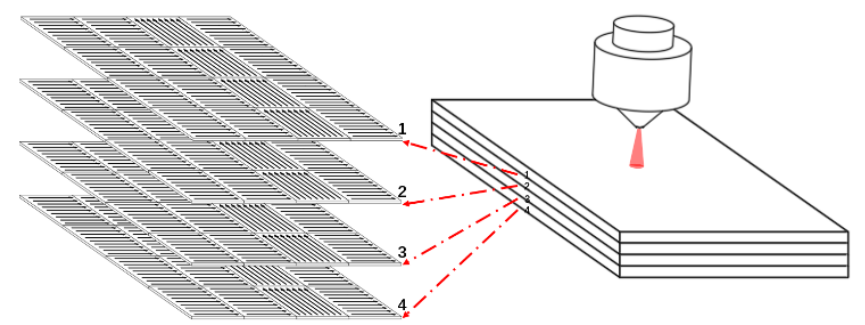

Fig. 1: Illustration of the AM scanning path pattern.

However, the residual stress will be accumulated during the manufacturing process due to inconsistent level of heating, which leads to undesirable distortion, cracks, delamination, and warpage [9-10]. There are many researches proposed to resolve above mentioned challenges. [11] proposed a topology optimization scheme to minimize the mean compliance and the part distortion. A thermo-elastic element-birth model is adopted in this method to better describe the AM process. Besides, a layer-bylayer thermo-elastic model was constructed and incorporated with the level set topology optimization method in to constraint the structural thermal stress [12]. Good results have been achieved in both approaches, though the computational cost is intensive. To ensure the built quality of the parts, [13] proposed a scanning path optimization method to against the residual stress/distortion. However, the optimized path obtained by the authors are complex, and hard to be adopted in real application.

In this work, a systemic metal AM oriented path optimization method, which could reduce the distortion during the manufacturing process, is proposed by us. First, a voxel-based methodology is 
employed to generate efficient Cartesian mesh of the printed part for finite element analysis. Instead of using full-scale thermal-elastic simulation, for the purpose of saving computational cost, a layer-by-layer inherent strain method is adopted to efficiently describe the complex physical behavior during the AM process. To avoid the mesh regeneration in the optimization iterations, the voxel-based Cartesian mesh is used to discretize the design domain of a given design domain. Then, a non-constraint optimization problem is formulated to find the optimal island scanning path in each print layer. In the end, a fan blade example is provided, which indicates the effectiveness of this method.

In practice, it is quite easy to set or adjust the island scanning path of the laser for the metal AM machine, and the non-constraint optimization model also ensures the robustness of the algorithm. The proposed method therefore could be broadly applied in metal AM.

\section{Main Idea:}

\section{Inherent strain method}

The physical model of the layer-by-layer type building process is simulated based on the inherent strain method, which is a couple of several linear elastic analyses. This method enables for a fast prediction of the distortions and residual stresses of the fabricated component. During the heating and cooling cycle of the metal AM processes, the total strain $\epsilon^{\text {tatal }}$ could be split into elastic strains $\epsilon^{\text {elastic }}$, thermal strains $\epsilon^{\text {thermal }}$, plastic strains $\epsilon^{\text {plastic }}$, phase transformation strains $\epsilon^{\text {phase }}$ and creep strains $\epsilon^{\text {creep }}$, and expressed as:

$$
\epsilon^{\text {tatal }}=\epsilon^{\text {elastic }}+\epsilon^{\text {thermal }}+\epsilon^{\text {plastic }}+\epsilon^{\text {phase }}+\epsilon^{\text {creep }}
$$

Recalling the balance of momentum equation for a quasi-static analysis, we arrive:

$$
\nabla \boldsymbol{\sigma}+\mathbf{b}=0
$$

where $b$ is the body force vector. When defining the inherent strain as: $\epsilon^{\text {ihs }}=\epsilon^{\text {thermal }}+\epsilon^{\text {plastic }}+\epsilon^{\text {phase }}+$ $\boldsymbol{\epsilon}^{\text {creep }}$, the Cauchy stress tensor $\boldsymbol{\sigma}$ is written as:

$$
\sigma=\mathbf{D}\left(\boldsymbol{\epsilon}^{\text {tatal }}-\epsilon^{\text {ihs }}\right)
$$

To reduce the computational cost, the components of the original inherent strain tensor $\epsilon^{\text {ins }}$ could be directly obtained by experimental calibration or high-fidelity simulation method [14]. By rotating the $\boldsymbol{\epsilon}^{\text {ihs }}$, the elemental inherent strain $\boldsymbol{\epsilon}^{*}{ }_{e}$ in each scanned island (0) is expressed as:

$$
\boldsymbol{\epsilon}^{*}{ }_{e}=\mathbf{R} \boldsymbol{\epsilon}^{\mathrm{ihs} 0} \mathbf{R}^{\mathrm{T}}
$$

where $\mathbf{R}$ is the xy-plane rotation matrix, which could be expressed as a function of the orientation angle $\vartheta_{e}$ :

$$
\boldsymbol{R}=\left[\begin{array}{ccc}
\cos \left(\vartheta_{e}\right) & \sin \left(\vartheta_{e}\right) & 0 \\
-\sin \left(\vartheta_{e}\right) & \cos \left(\vartheta_{e}\right) & 0 \\
0 & 0 & 1
\end{array}\right]
$$

Note that there is a mapping relationship between the island-wise orientation angle $\boldsymbol{\theta}$ and element-wise orientation angle $\boldsymbol{\vartheta}$ :

$$
\boldsymbol{\vartheta}=\mathbf{M} \boldsymbol{\theta}
$$

the $\mathbf{M}$ is mapping matrix with $\mathrm{Nel} \times \mathrm{Nd}$ dimensions, where $\mathrm{Nel}$ is the total number of finite elements in this model, and $\mathrm{Nd}$ is the total number of design variables.

\section{Metal additive manufacturing process model}

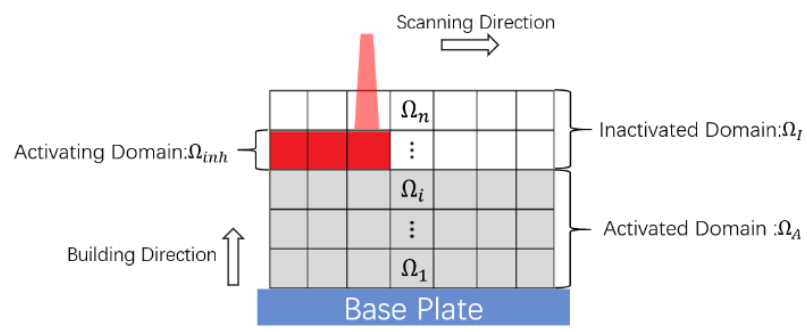

Fig. 2: Illustration of the AM process model. 
As shown in the Fig. 2, the domain $\Omega$ is divided into m layers with a fixed thickness along the building direction. Each layer is defined by $\Omega_{\mathrm{i}}$ and $1 \leq \mathrm{i} \leq \mathrm{m}$. The domain $\Omega$ is composed by three subdomains, where represented by $\Omega_{\mathrm{ihs}}, \Omega_{\mathrm{act}}$, and $\Omega_{\text {pass }} . \Omega_{\mathrm{ihs}}$ is the layer to be printed and to apply the inherent strain, $\Omega_{\text {act }}$ is the already printed layers, and $\Omega_{\text {pass }}$ is the unprinted layers. It is reasonable to assume the inherent strain $\epsilon^{*}$ at the domain $\Omega_{\text {ins }}$ contributes to the distortion in the rest part of the substrate. Within above assumptions, the displacements $\mathbf{U}^{\mathrm{i}}$ of printing stage i are calculated as follows:

$$
\mathbf{K}^{\mathrm{i}} \mathbf{U}^{\mathrm{i}}=\mathbf{F}^{\mathrm{i}}
$$

where

and

$$
\mathbf{F}^{\mathrm{i}}=\sum_{\mathrm{e}=1}^{\mathrm{Nel}}\left(\vartheta_{\mathrm{e}}^{\mathrm{i}} \mathbf{L}_{\mathrm{e}}^{\mathrm{T}} \int \mathbf{B}^{\mathrm{T}} \mathbf{D}_{\mathrm{e}} \epsilon^{*} \mathrm{~d} \Omega_{\mathrm{e}}\right)
$$

$$
\mathbf{K}^{\mathrm{i}}=\sum_{\mathrm{i}=1}^{\mathrm{m}}\left(\sum_{\mathrm{e}=1}^{\mathrm{Nel}}\left(\delta_{\mathrm{e}}^{\mathrm{i}} \mathbf{L}_{\mathrm{e}}^{\mathrm{T}} \mathbf{K}_{\mathrm{e}} \mathbf{L}_{\mathrm{e}}\right)\right)
$$

$\vartheta_{\mathrm{e}}^{\mathrm{i}}$ and $\delta_{\mathrm{e}}^{\mathrm{i}}$ are two flag variables to indicate the domain $\Omega_{\mathrm{ihs}}$ and $\Omega_{\mathrm{act}}$, the matrix $\mathbf{L}_{\mathrm{e}}$ gathers the nodal displacements of the eth element ( $\mathbf{u}_{\mathrm{e}}$ ) from the global displacement vector (U) satisfying $\mathbf{u}_{\mathrm{e}}=\mathbf{L}_{\mathrm{e}} \mathbf{U}$, and $\mathbf{K}_{\mathrm{e}}$ is the elemental stiffness matrix which is calculated as follows:

$$
\mathbf{K}_{\mathrm{e}}=\int \mathbf{B}^{\mathrm{T}} \mathbf{D}_{\mathrm{e}} \mathbf{B} \mathrm{d} \Omega_{\mathrm{e}}
$$

where $\mathbf{B}$ is the strain-displacement matrix, and $\mathbf{D}_{0}$ is the constitutive matrix for the solid material. The part-scale displacement for a specific printing stage i is determined as a sum of the displacements all layers printed so far:

$$
\mathbf{U}=\sum_{\mathrm{i}=1}^{\mathrm{m}} \mathbf{U}^{\mathrm{i}}
$$

The resulting displacements of the AM process simulation are shown in 0.

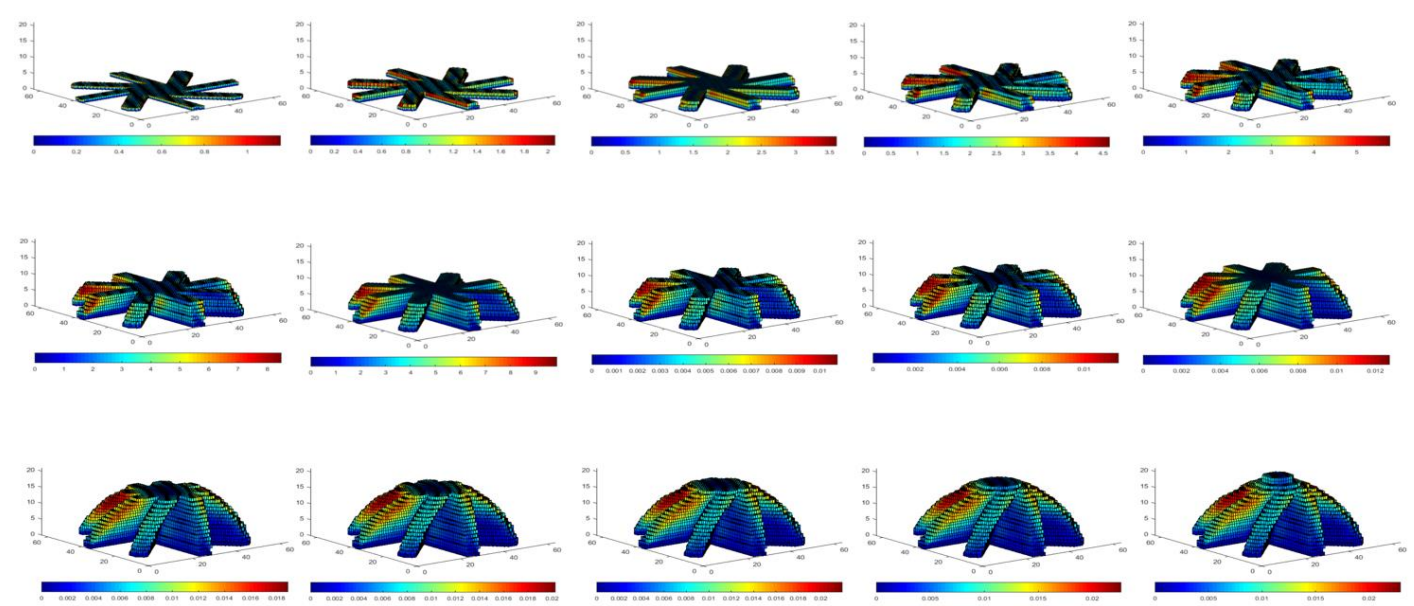

Fig. 3: Deformation of the fan blade during the metal AM process.

\section{Non-constraint optimization problem formulation}

For the optimization problem, the objective function is to minimize the structural distortion under the inherent strain load. The mathematical formulation of this optimization problem can be expressed as follows: 


$$
\left\{\begin{array}{c}
\text { find: } \boldsymbol{\theta} \\
\text { minimize: } \mathrm{J}=\frac{\sum_{j=1}^{N d o f}\left(U_{j}\right)^{2}}{\sum_{j=1}^{N d o f}\left(U_{j}^{0}\right)^{2}} \\
\text { subject to: }\left\{\begin{array}{c}
\mathbf{K}^{\mathrm{i}} \mathbf{u}^{\mathrm{i}}=\mathbf{F}^{\mathrm{i}}(\mathrm{i}=1,2, \ldots, \mathrm{m}) \\
0^{\circ} \leq \forall \theta \leq 90^{\circ}
\end{array}\right.
\end{array}\right.
$$

The deformation can be measured through $\sum_{j=1}^{N d o f}\left(U_{j}\right)^{2}$, where $j=1,2, \ldots N$ dof is the degrees of freedom with which displacements caused by the inherent strain load, and the term $\sum_{j=1}^{N d o f}\left(U_{j}^{0}\right)^{2}$ indicates the distortion of the part with default scanning path pattern. The Method of Moving Asymptotes (MMA) will be adopted to solve the optimization problem, and the process of this method is shown in 0 .

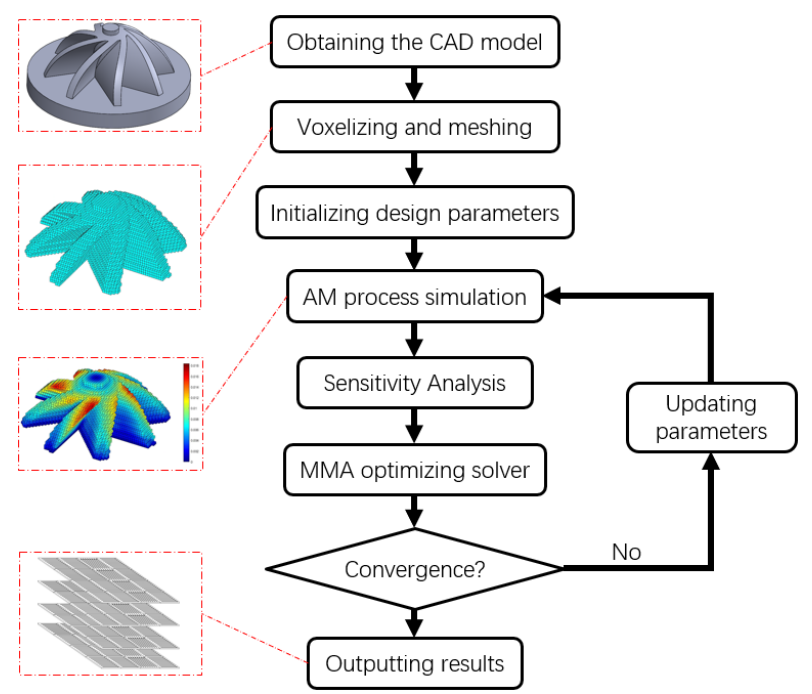

Fig. 4: The process of the proposed method.

Case study

The numerical example is a fan blade used in mining machinery, and its voxelized mesh model is shown in 0 . The build direction is down-top, and the design domain is divided into $\mathrm{m}=20$ layers, with a layer thickness of $1 \mathrm{~mm}$ in the building direction. In each print layer, there are $8 \times 8$ scanning islands.
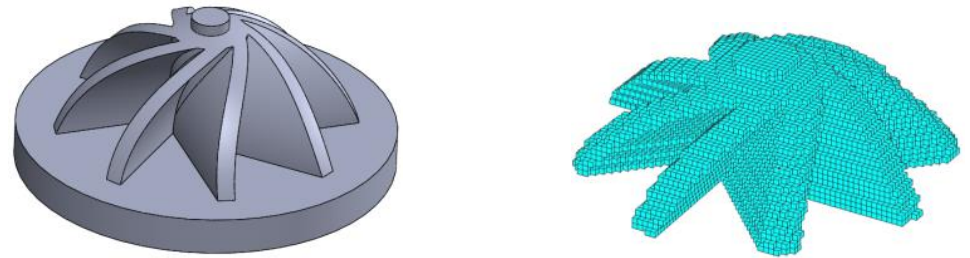

Fig. 5: The CAD model for the fan blade (left), and the voxelized finite element model (right).

Firstly, this part is simulated by all scanning islands with the same scanning orientation $180^{\circ} .0$ presents the simulation result containing the part-scale distortion distribution for the as-fabricated part. Since the scanning orientation is the same in each layer, larger distortions are concentrated at the upper outer edges of two blades, and the maximum distortion is $0.024 \mathrm{~mm}$. 

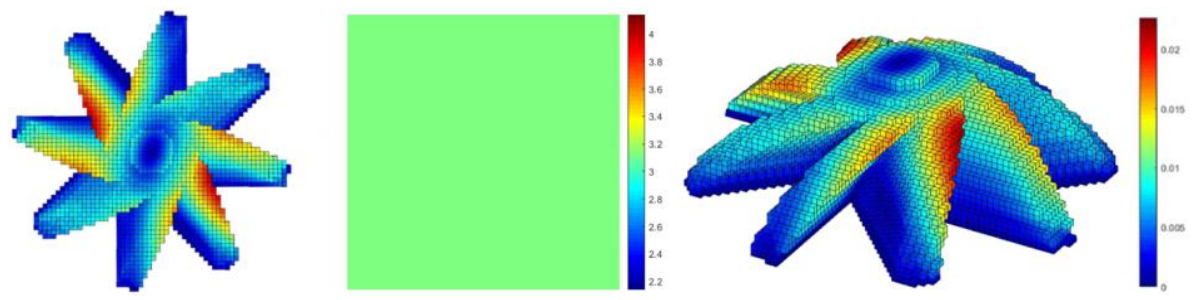

Fig 6: The distortions for the part printed without scanning path optimization.

Fig. 7 presents the part-scale distortion distribution for the as-fabricated part with optimized scanning path. Recalling that in our optimization problem formulation, the objective is to reduce the part-scale distortion, which is defined as the sum of global nodal displacements. Therefore, more even residual distortion distribution could be observed in the part, although the higher distortions are still distributed at outer edges of blades. Specifically, the part printed by optimized scanning path exhibits apparently smaller deformation (i.e., the maximum distortion is $\mathrm{u}_{\max }=0.012 \mathrm{~mm}$ ) compared to the part in 0 , $\mathrm{u}_{\max }=$ $0.024 \mathrm{~mm}$.
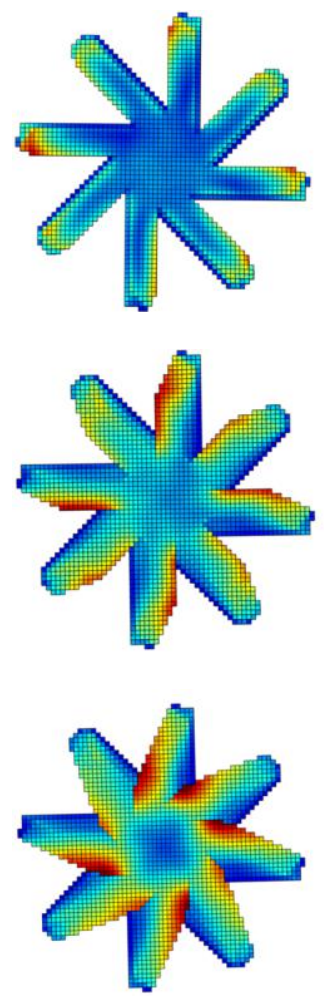

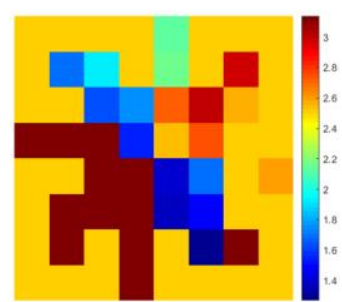

(a)

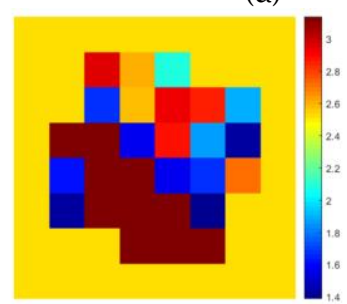

(b)

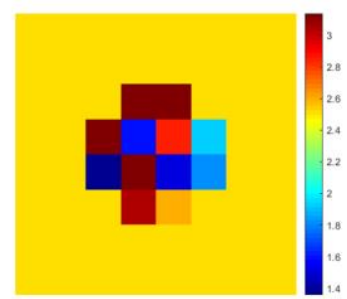

(c)
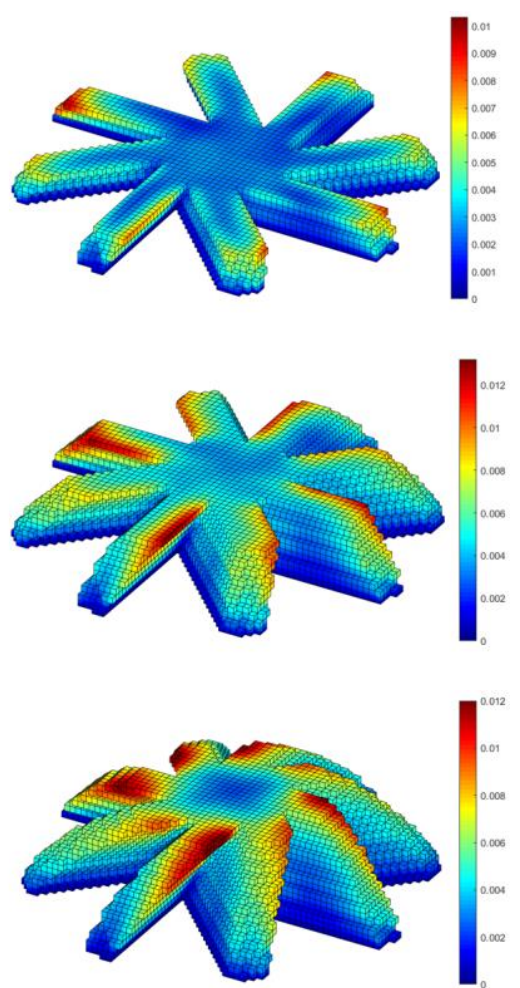

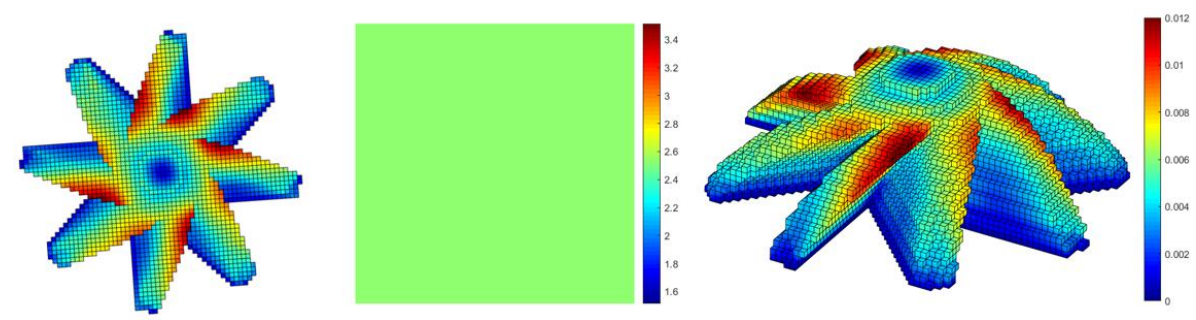

(d)

Fig. 7: The distortions for the part with optimized scanning path: (a) layer 5, (b) layer 10, (c) layer 15, and (d) layer 20.

The convergence history curve for the optimization process is proposed in 0 . As the number of iterations increases, the objective value is reduced and finally approached to a fixed value. Again, as we mentioned before, this optimization problem is non-constrained and the design variables are independent with each other, that is the reason why a smooth and sufficient convergence could be obtained within only 30 iterations.

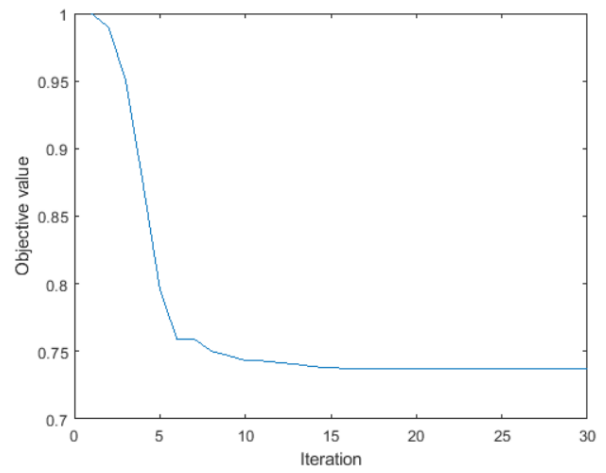

Fig. 8: The convergence history.

\section{Conclusion}

The proposed method could successfully reduce the distortion induced by the metal AM process through optimizing the laser scanning path. A typical AM oriented part is studied to examine the performance of this method. We compare the part-scale residual distortion distribution between the parts printed by different scanning path. It is found that the scanning path plays an important role for distortion minimization, and the part printed by the optimized scanning path exhibits better performances (smaller distortion). Besides, a fast and stable convergence curve shown in 0 also indicates the efficiency of the proposed method. Thus, this method is possible to ensure manufacturability of AM builds.

Note that although the proposed methodology obtains a good result within the numerical simulation, further demonstration of the efficiency of the proposed method is still needed. In the future, the experiment will be supplemented.

\section{References:}

[1] Liu, J.; Gaynor, A. T.; Chen, S.; Kang, Z.; Suresh, K.; Takezawa, A.; Li, L.; Kato, J.; Tang, J.; Wang, C. C. L.; Cheng, L.; Liang, X.; To, A. C.: Current and future trends in topology optimization for additive manufacturing. Structural and Multidisciplinary Optimization, 57(6), 2018, 2457-2483. https://doi.org/10.1007/s00158-018-1994-3

[2] Liu, J.; Ma, Y.: A survey of manufacturing-oriented topology optimization methods. Advances in Engineering Software, 100, 2016, 161-175. https://doi.org/10.1016/j.-advengsoft.2016.07.017 
[3] Huang, J.; Chen, Q.; Jiang, H.; Zou, B.; Li, L.; Liu, J.; Yu, H.: A survey of design methods for material extrusion polymer 3D printing. Virtual and Physical Prototyping, 15(2), 2020, 148-162. https://doi.org/10.1080/17452759.2019.1708027

[4] Xu, S.; Huang, J.; Liu, J.; Ma, Y.: Topology Optimization for FDM Parts Considering the Hybrid Deposition Path Pattern. Micromachines, 11(8), 2020, 709. https://doi.org/10.3390/-mi11080709

[5] Liu, J.; Ma, Y. S.: 3D level-set topology optimization: a machining feature-based approach. Structural and Multidisciplinary Optimization, 52(3), 2015, 563-582. https://doi.org/10.1007/s00158-015-1263-7

[6] Liu, J.: Guidelines for AM part consolidation. Virtual and Physical Prototyping, 11(2), 2016, 133141. https://doi.org/10.1080/17452759.2016.1175154

[7] Frazier, W. E.: Metal additive manufacturing: a review. Journal of Materials Engineering and performance, 23(6), 2014, 1917-1928. https://doi.org/10.1007/s11665-014-0958-Z

[8] King, W. E.; Anderson, A. T.; Ferencz, R. M.; Hodge, N. E.; Kamath, C.; Khairallah, S. A.; Rubenchik, A. M.: Laser powder bed fusion additive manufacturing of metals; physics, computational, and materials challenges. Applied Physics Reviews, 2(4), 2015, 041304. https://doi.org/10.1063/1.4937809

[9] Cheng, L.; Liang, X.; Bai, J.; Chen, Q.; Lemon, J.; To, A.: On utilizing topology optimization to design support structure to prevent residual stress induced build failure in laser powder bed metal additive manufacturing. Additive Manufacturing, 27, 2019, 290-304. https://doi.org/10.1016/j.addma.2019.03.001

[10] Cheng, L.; To, A.: Part-scale build orientation optimization for minimizing residual stress and support volume for metal additive manufacturing: Theory and experimental validation. Computer-Aided Design, 113, 2019, 1-23. https://doi.org/10.1016/j.cad.2019.03.004

[11] Wildman, R. A.; Gaynor, A. T.: Topology Optimization for Reducing Additive Manufacturing Processing Distortions (No. ARL-TR-8242). Weapons and Materials Research Directorate, US Army Research Laboratory Aberdeen Proving Ground United States, 2017.

[12] Allaire, G.; Jakabčin, L.: Taking into account thermal residual stresses in topology optimization of structures built by additive manufacturing. Mathematical Models and Methods in Applied Sciences, 28(12), 2018, 2313-2366. https://doi.org/10.1142/S0218202518500501

[13] Chen, Q.; Liu, J.; Liang, X.; To, A. C.: A level-set based continuous scanning path optimization method for reducing residual stress and deformation in metal additive manufacturing. Computer Methods in Applied Mechanics and Engineering, 360, 2020, 112719. https://doi.org/10.1016/j.cma.2019.112719

[14] Bugatti, M.; Semeraro, Q.: Limitations of the inherent strain method in simulating powder bed fusion processes. Additive Manufacturing, 23, 2018, 329-346. https://doi.org/10.1016/j.addma.2018.05.041 